\title{
In Vitro Simulation of Human Digestion: Chemical and Mechanical Behavior
}

\author{
Sara Cascone ${ }^{1,{ }^{*}}$, Annalisa Dalmoro ${ }^{2}$, Gaetano Lamberti ${ }^{1}$, Giuseppe Titomanlio ${ }^{1}$, \\ Matteo d'Amore 2 , and Anna Angela Barba² \\ ${ }^{1}$ Department of Industrial Engineering, Via Giovanni Paolo II 132, University of Salerno, 84084 Fisciano (SA), Italy \\ ${ }^{2}$ Department of Pharmacy, Via Giovanni Paolo II 132, University of Salerno, 84084 Fisciano (SA), Italy
}

e-mail: scascone@unisa.it

\begin{abstract}
The drug release pattern from an orally administrated pharmaceutical dosage form can be significantly affected by simultaneous food intake and drug administration due to the changes in the gastrointestinal physiology, in particular because of the $\mathrm{pH}$ profile evolution and of the mixing conditions. In this work, the release from a commercial diclofenac tablet subjected to the $\mathrm{pH}$ conditions experienced by the gastrointestinal tract in fed conditions was analyzed and then compared with the release pattern obtained using a conventional dissolution method. The tablets behaved differently because of the partial dissolution of the coating due to high $\mathrm{pH}$ values in the first stage of the dissolution method, which takes into account the fed-state conditions. Tablets composed of a homemade enteric polymer loaded with diclofenac overcame this drawback. Moreover, an in vitro device mimicking peristaltic contractions in the stomach was proposed, and the release pattern from commercial tablets was compared with that obtained under conventional dissolution conditions. The release pattern was strongly influenced by the hydrodynamics. The two experimental setups $(\mathrm{pH}$ and peristalsis simulations) demonstrate that a gastrointestinal reproduction closer to the real physiology is necessary to achieve an accurate and reliable prediction of the behavior of pharmaceuticals.
\end{abstract}

KEYWORDS: In vitro device; pH evolution; peristaltic waves; drug release; dissolution.

\section{INTRODUCTION}

$t$ has long been recognized that the presence of food may influence drug absorption as a result of physiological changes in the gastrointestinal tract due to the interactions between food particles and drug molecules $(1,2)$. Thus, drug bioavailability can be significantly affected by concomitant food intake and drug administration due to variations of the drug absorption rate and extent, and to the changes in gastrointestinal physiology (luminal changes, gastric motility, and transit time variations) $(3,4)$. To understand the influence of food on oral administration, it is critical to analyze all the processes involved during digestion under fasted and fed conditions.

Modeling of food and food processes is an important task in food engineering, and several approaches are possible (5). For example, the heat and mass transport equations can be coupled with a computational fluid dynamics (CFD) tool to simulate gastric velocity fields (6, 7) or fluid motion in the oral cavity (8), with biochemical reactions to describe the enzymatic transformations or soluble solid loss due to a decreasing $\mathrm{pH}$ (9), or with mechanical effects (5). Moreover, to evaluate the heat and mass transfer phenomena occurring during food processing, more complex approaches can be used, such as a multiscale modeling, where phenomena happening on both a very small scale and a macroscale are coupled to describe the global process (10). Beside its scientific relevance, mathematical modeling of food transformation is particularly challenging due to the complexity of involved phenomena during the processing of food; its temperature, composition, structure, and texture can change leading to variations of its physical properties. Thus, during a realistic simulation, the model has to take into account a dynamic estimation of the food physical properties that continue to change during the process (11).

To study the structural changes, digestibility, and release of food components during digestion, in vitro digestion models are the most used tools. Several researchers have used these methods, and the most widely tested food samples have been plants, meats, fish, dairy, and emulsion-based foods (12). Many differences are evident in these studies, depending on the specific food component analyzed, the nature of the food matrix, and the complexity of the simulating system used. In fact,

* Corresponding author. 
simulation of food digestion can be performed using simple methods, such as the $\mathrm{pH}$-drop, which has been used to predict the protein digestibility of foods (13) by measuring the $\mathrm{pH}$ decrease during peptide release, and the $\mathrm{pH}$-stat, which measures the amount of $\mathrm{NaOH}$ needed to maintain constant $\mathrm{pH}$. Another simple method used to evaluate the digestibility of starch from processed foods is the colorimetric technique. This is based on the colorimetric analysis of glucose or maltose liberated from starch by enzymes (14). On the other hand, devices that are more complex have been proposed to simulate the hydrodynamics and biochemical conditions in the gastrointestinal (GI) tract. To simulate the kinetics of a food digestion process, Chen et al. (15) proposed an in vitro digestion device consisting of a jacketed glass vessel and a spherical Teflon probe controlled by a texture analyzer. The probe can be moved up and down under the control of the texture analyzer creating a flow pattern similar to that of the contraction waves of the stomach wall. The in vitro digestion model has been tested on peanut particles showing the reliability and ease of control of the device. With the aim of studying the effect of viscosity in transport phenomena during the digestion in the small intestine, a small intestinal model (SIM) was developed (16) taking into account the intestinal physiology. The model is composed of two compartments, the lumen side, represented by an inner tube, and the recipient side, separated by a semipermeable membrane. Segmentation motion along the intestine is reproduced by inflation and deflation of rubber cuffs around the tube, alternatively applying pneumatically compressed air and vacuum. A complete and detailed in vitro model was developed by the Nutrition and Food Research Institute (TNO, the Netherlands) (17). The model consists of four chambers simulating the stomach, duodenum, jejunum, and ileum. Each chamber is composed of a glass jacket equipped with a flexible wall inside. A fluid is pumped between the glass wall of the chamber and the flexible wall to control the temperature in the inner portion and mix the internal contents by alternate compression and relaxation of the flexible wall. The jejunal and ileal compartments are equipped with hollow fibers to simulate the absorption of the digestion products. This model allows the reproduction of the $\mathrm{pH}$ and bile evolutions and the absorption of glucose found in vivo. Wickham and Faulks (18) proposed the Dynamic Gastric Model (DGM), which provides different stages including a pretreatment necessary to reproduce the chewing of foods. The first stage simulates the main body of the stomach (the fundus), characterized by an inhomogeneous mixing behavior and by the addition of acid and enzymes. The second stage of the model simulates the antrum, which is a high-shear zone. When the foodstuff achieves a size sufficient to mimic the physiological one, a valve (the pylorus) is opened and the plunger is pushed upward so that the processed food can leave the chamber. This model accurately reproduces the shear stresses inside the stomach. More recently, Kong and Singh (19) presented the Human Gastric Simulator (HGS) model to study gastric digestion of foods. The HGS is designed to simulate the continuous peristaltic movement of the stomach walls, with amplitude and frequency of contraction forces similar to those reported in vivo. The precise control of gastric secretion and emptying and the adjustable mechanical forces in the HGS provide a useful tool to study the transformation of food constituents under simulated physiological conditions.

Thus, to reproduce the digestion process and to propose an in vitro device that simulates the gastrointestinal tract, food presence and fed conditions have to be taken into account (20). In addition, in vitro food digestion models are used increasingly in the pharmaceutical industry to screen the digestibility of products with different compositions and structures (21). Therefore, the reproduction of realistic gastrointestinal conditions during the dissolution test plays an important role in the oral delivery of solid dosage forms and is necessary to evaluate the critical parameters that affect the process (22). In fact, the release from solid dosage forms is mostly affected by the gastrointestinal conditions since the biochemical and mechanical conditions experienced by pharmaceuticals influence the release kinetics and thus the drug concentration in the entire body.

The aim of this work was to evaluate the influence of food presence on drug release by taking into account two key physiological factors that are affected by food intake, the evolution of the $\mathrm{pH}$ profile in the gastrointestinal tract and the mixing conditions in the stomach. To this purpose, two devices were designed and built to simulate stomach behavior that is closer to real physiology and to obtain more reliable drug release profiles.

\section{MATERIALS AND METHODS Materials}

Extended-release commercial tablets of diclofenac (DOC Generici s.r.l., Milan, Italy) were used for the dissolution tests. Each tablet contains $100 \mathrm{mg}$ of the drug. The tablets were coated to resist the low $\mathrm{pH}$ in the stomach and release in a neutral environment such as the intestines. Release of drug in the stomach and upper small intestine is undesirable because this leads to premature absorption and consequent drug wastage as well as possible systemic side effects. These commercial tablets were chosen to

Dissolution 
evaluate if a pharmaceutical dosage form designed to pass through the stomach without dissolving actually has the desired release profile, even in simulated physiological conditions. Moreover, diclofenac is a well-known and studied drug and is used extensively, thus it could be considered a model drug. For the release studies based on the homemade enteric tablets of diclofenac, matrices with a total weight of $280 \pm 5 \mathrm{mg}$ were composed of diclofenac $(25 \% \mathrm{w} / \mathrm{w})$ and one excipient with enteric features $(75 \%$ $w / w)$. The excipient was a methyl methacrylate/acrylic acid (MMA/AA) copolymer synthesized with 75/25 (v/v) of MMA/AA (23). Diclofenac was purchased from Sigma Aldrich srl, Milan, Italy.

To prepare the buffer solutions used during the conventional dissolution tests, $\mathrm{HCl}(37 \% \mathrm{w} / \mathrm{w}$ solution) and sodium phosphate tribasic dodecahydrate were purchased from Sigma-Aldrich, Milan, Italy. Hydrochloric acid and sodium hydroxide solutions were used to adjust the $\mathrm{pH}$ values of the dissolution medium during the simulation of the gastrointestinal tract $\mathrm{pH}$ evolution.

\section{Conventional Human Digestion Simulation}

To reproduce the gastrointestinal environment, different media were used during the dissolution tests. During the first two hours, a solution at $\mathrm{pH} 1(6.25 \mathrm{~mL}$ of $\mathrm{HCl}$ diluted to $750 \mathrm{~mL}$ with deionized water) was used to reproduce the acidic environment of the stomach, according to the USP recommendation (24). After two hours, which represents the average residence time in the stomach (24), the solution was neutralized to $\mathrm{pH} 6.8$ to reproduce the almost neutral intestinal environment. The neutralization was achieved by adding $250 \mathrm{~mL}$ of deionized water containing $19 \mathrm{~g}$ of sodium phosphate tribasic dodecahydrate to the previous solution. Therefore, for the conventional dissolution release, a two-step $\mathrm{pH}$ evolution was used, as shown in the left axis of Figure 1. The dissolution tests were performed with USP Apparatus 2 (AT7 Smart, Sotax, Allschwil, Switzerland). During all the tests, the medium was kept at $37{ }^{\circ} \mathrm{C}$ and stirred by paddle at a constant rotation speed of $100 \mathrm{rpm}$, which results in a high-intensity shear force exerted on the fluid (25). In these conditions, it was possible to compare the USP stirring effect on pharmaceuticals with peristaltic movement. During the dissolution tests, 1-mL samples of dissolution medium were taken and analyzed by HPLC to evaluate the drug concentration profile. During the test, about 30 samples were withdrawn from the dissolution medium, which had a total volume of $1 \mathrm{~L}$. Thus, the volume decrease during the test did not influence the release profile. Moreover, in the evaluation of the drug amount in the dissolution medium, the volume decrease was accounted for by

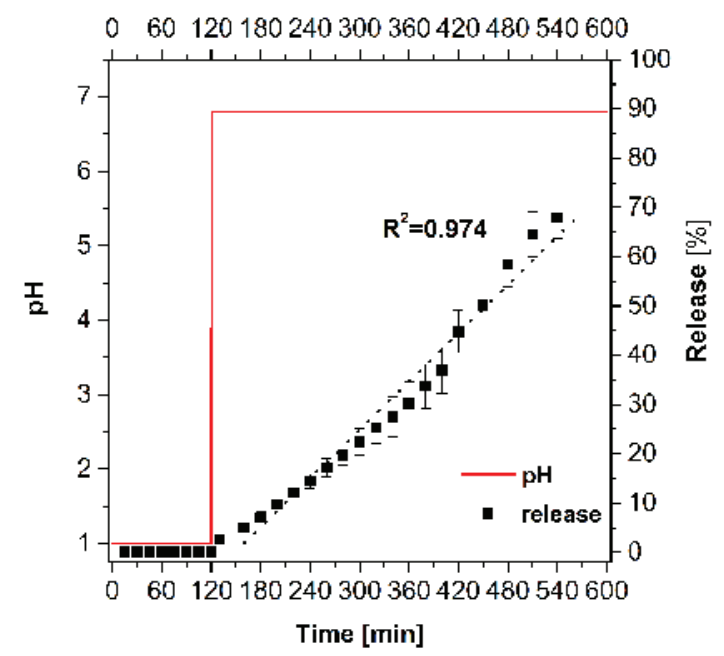

Figure 1. $\mathrm{pH}$ evolution during the conventional gastrointestinal environment simulation (left axis) and drug release from a commercial diclofenac extended-release tablet (right axis). The dotted line is the linear regression of the experimental data during the dissolution in the intestine.

multiplying the concentration measured and the actual dissolution volume. The diclofenac concentration was measured, and the percentage release was evaluated according to the following expression:

$$
\% \text { Release }=\frac{\text { drug released } \text { in the dissolution medium }}{\text { drug initially contained in the tablet }} \times 100
$$

This release profile was used as the reference release pattern for comparison with the results obtained from the other dissolution tests. All experiments were conducted in triplicate to ensure the reproducibility of the data, which are expressed as average values. The error bars represent the standard deviations among the experiments.

\section{pH Effect on Human Digestion}

The $\mathrm{pH}$ in the stomach is higher in fed conditions than in fasted conditions (4), and its value depends on the $\mathrm{pH}$ and the buffer capacity of the food. After food ingestion, the $\mathrm{pH}$ decreases due to acid secretions (26). Thus, the $\mathrm{pH}$ in the stomach is not always 1 , as adopted by the conventional dissolution method. Instead, just before a meal, the $\mathrm{pH}$ is about 4.8 and starts to decrease after the meal (27). The physiological $\mathrm{pH}$ evolution in the gastrointestinal tract (the real behavior) is reported in Figure 2 as a black dotted line. During the first two hours, the $\mathrm{pH}$ decreases from 4.8 to about 1.7, then remains at a constant value of 6.8 when the stomach contents are transferred to the intestine. In the dissolution tests performed to evaluate the $\mathrm{pH}$ effect on drug release, the initial $\mathrm{pH} 4.8$ buffer was prepared by mixing deionized water containing $16.6 \mathrm{~mL}$ of $\mathrm{HCl}$ and $38 \mathrm{~g}$ of sodium phosphate tribasic dodecahydrate and 
dissolving to a volume of $500 \mathrm{~mL}$. To decrease the medium $\mathrm{pH}$ to 1 during the dissolution, two peristaltic pumps were used to dose acidic ( $83 \mathrm{~mL}$ of $\mathrm{HCl}$ diluted in 500 $\mathrm{mL}$ of deionized water) or basic $(40 \mathrm{~g}$ of $\mathrm{NaOH}$ dissolved in $500 \mathrm{~mL}$ of deionized water) solutions. The $\mathrm{pH}$ control was performed using a glass $\mathrm{pH}$ probe (RS-Instruments, Italy, cod. 205-075) connected to a data acquisition board (National Instruments DAQ NI PCl-6031) and dedicated software to drive the pump actions on the basis of the error between the set point $\mathrm{pH}$ value and the measured value, according to a previously developed method (28). After two hours, the sudden increase in $\mathrm{pH}$ was achieved by adding a solution of $0.4 \mathrm{~g} \mathrm{NaOH}$ in $25 \mathrm{~mL}$ deionized water, in addition to the dosage of the basic solution controlled by the pumps. The measured $\mathrm{pH}$ evolution is shown in Figure 2 as a continuous grey line (left axis). The set point $\mathrm{pH}$ evolution in the dissolution medium is well approximated. As in conventional tests, about 30 samples of medium were taken and analyzed by an HPLC method during the dissolution to evaluate the release profile.

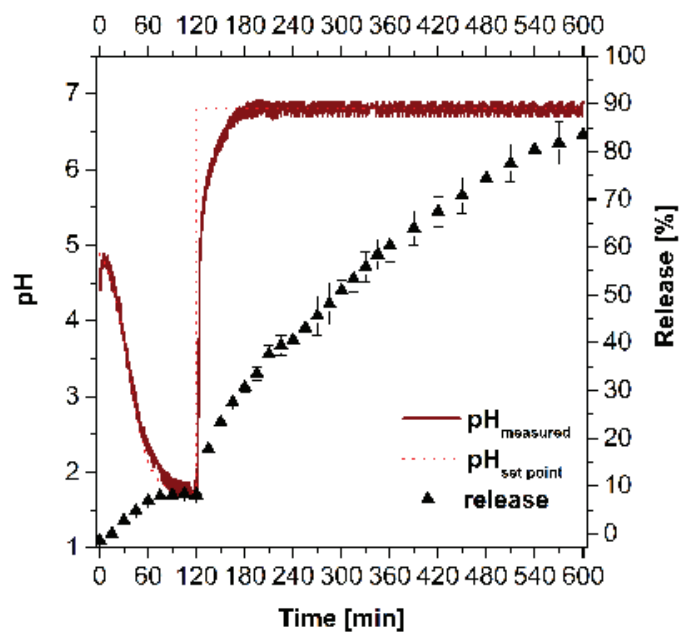

Figure 2. Physiological $\mathrm{pH}$ evolution after a meal in the gastrointestinal environment (dotted red line) (Adapted with permission from ref 27. Copyright 1990 The Nutrition Society.) and its reproduction in the proposed model (continuous line) on the left axis. Drug release from a commercial extended-release tablet of diclofenac on right axis.

\section{Mechanical Effect on Human Digestion}

To reproduce the stomach mechanical history, an in vitro device was developed. The stomach chamber was made of latex, which is compressible and elastic to guarantee the appropriate deformation when the contractions are applied. The stomach chamber is larger at the top and thinner at the bottom (oval shaped) to mimic the real stomach anatomy, with a total volume of $1 \mathrm{~L}$. To mimic real physiology, the simulated peristaltic waves have to start in the antral zone at a rate of three contractions per minute. The magnitude of the contractions increases from the top to the bottom, thus the maximum strength is realized in correspondence with the pylorus position in the stomach. Therefore, in the in vitro device, contractions were applied in three different positions, each ensured by a guide, as shown in Figure 3 . Figure $3 \mathrm{~A}$ is a photograph of the proposed artificial stomach. The contraction positions are guaranteed by guides, and the peristaltic movement is realized by three belts that connect the stomach to a rotating shaft. Figure $3 \mathrm{~B}$ is a schematic overhead view of the system. The shaft is equipped with three pairs of iron bars, represented in light gray. Each pair of bars is spaced by a support that permits the sliding of the belt; this support is represented by black circles on the bar. The position of the support determines the strength of the contraction, because the greater the distance between support and the center of the shaft, the more stretched are the belts and the stronger is the contraction. As can be seen from the schematic in Figure 3B, contraction 1, related to the upper part of the stomach, has its support near the center of the shaft, which means that when cam 1 is opposite the stomach, there is a light shrinking of the belt connected, represented by the grey line connected to the stomach. In contrast, the support located at position 3 is a significant distance from the center of the shaft, so that when cam 3 rotates in the opposite direction with respect to the stomach, shrinking is generated, giving a strong stomach contraction. To mimic real physiology, the contractions were designed to be stronger at the bottom

A

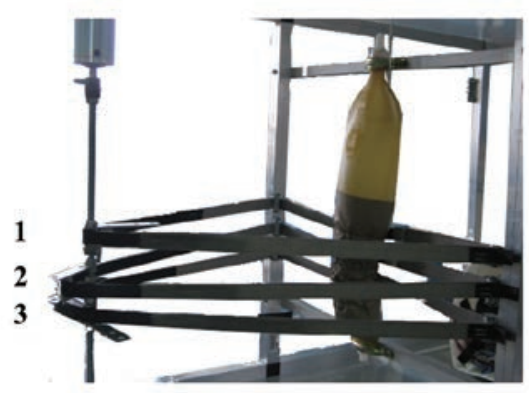

B

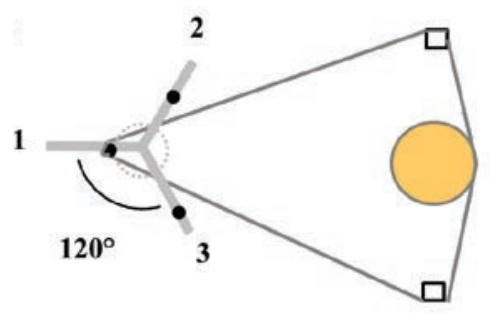

Figure 3. (A) Photograph of the proposed in vitro device. (B) Schematic of the overhead view. The camshaft system is connected to the artificial stomach by belts, which go around the stomach, and according to the movement of the cams, shrink the stomach. 
than in the upper part. To reproduce the frequency of three contractions per minute, each contraction was designed to occur $20 \mathrm{sec}$ after the previous one, rotating the iron bars $120^{\circ}$ with respect to the upper one (Figure 3), and setting a shaft rotational rate of $1 \mathrm{rpm}$. The stomach contents were kept at a temperature of $37{ }^{\circ} \mathrm{C}$ during all tests. In this study, stomach empting was not considered because it actually influences only the dissolution volume without any relevant results on the release kinetics, which is influenced mainly by the mechanical stresses. To compare the results obtained from the conventional dissolution method and from the stomach simulation, and thus to isolate the mechanical effect on release profile, the $\mathrm{pH}$ in the artificial stomach was kept at 1 as in the conventional dissolution method. The dissolution medium was fed into the stomach, and when the tablet was inserted in the device, the experiment was started. After two hours, the entire stomach contents were transferred to Apparatus 2, neutralizing the dissolution medium to simulate the passage into the intestine. As in the previous dissolution tests, to measure the drug concentration released in the medium, 1- $\mathrm{mL}$ samples of the dissolution medium were withdrawn. All the experiments were conducted in triplicate to ensure reproducibility of the data, which are expressed as average values with standard deviations.

\section{Analytical Method}

High performance liquid chromatography (HPLCAgilent, 1200 Infinity series) was used to measure drug concentration in the samples. Analyses were performed with a Nucleodur 100-5 C18ec column, $150 \times 4.6 \mathrm{~mm}$ (Macherey-Nagel), an eluent of 48:52 acetonitrile:0.2\% acetic acid $(\mathrm{w} / \mathrm{w})$ in water, a flow rate of $2 \mathrm{~mL} / \mathrm{min}$, a temperature of $30^{\circ} \mathrm{C}$, a wavelength of $280 \mathrm{~nm}$, and an injection volume of $5 \mu \mathrm{L}$. The elution time of diclofenac was about 6 min.

\section{RESULTS AND DISCUSSION}

\section{Testing of Commercial Tablets by Varying pH}

The drug release of a commercial diclofenac extendedrelease tablet was evaluated using the conventional twostep $\mathrm{pH}$ dissolution method. Results are reported in Figure 1 (right axis). Drug release is negligible during the first two hours, in which the dissolution medium $\mathrm{pH}$ is acidic. During the first stage, the coating of the commercial tablet, which is designed to withstand a very acidic environment, was not attacked by the dissolution medium and the tablet was not damaged, preserving the drug entrapped in the matrix. When the dissolution medium was neutralized to a $\mathrm{pH}$ of 6.8 , the tablet released $70 \%$ of the drug in $9 \mathrm{~h}$ of dissolution, exhibiting almost linear kinetics. In fact, linear regression of the experimental data during the intestinal dissolution shows a good regression factor (Figure 1). This is the ideal behavior that an entericcoated tablet follows when ingested in the human body. As mentioned above, the real physiological $\mathrm{pH}$ evolution after food intake is completely different. For this reason, the release profile of the same commercial tablet was evaluated according to the modified $\mathrm{pH}$ evolution, also taking into account the effect of fed conditions. Results of the dissolution test are shown in Figure 2 (right axis). The release pattern is completely different from that of the previous one. Under these conditions, the tablet started to release the drug at the beginning of the dissolution test and continued during the first stage of dissolution. This behavior occurs because the dissolution medium is not as acidic as the conventional dissolution medium. When the tablet is immersed in the medium, it is subjected to a higher $\mathrm{pH}$, which attacks the tablet coating and leads to an unexpected drug release. Due to the variation of the first step conditions, the release pattern is different even during the second neutral step. Indeed, the release profile is not linear, because the dissolution of the coating in the initial step changes the release kinetics and leads to a different amount of drug release. With this dissolution method, the tablet releases about $80 \%$ of the drug after $9 \mathrm{~h}$ of dissolution, with faster kinetics than in the conventional method. In fact, a comparison of intermediate values of percentage drug release shows that the pharmaceutical form releases drug in a completely different manner. After $5 \mathrm{~h}$ of dissolution, the tablet tested according the conventional method releases about $20 \%$ of drug, and after $6 \mathrm{~h}$, it releases $30 \%$ of drug. Figure 2 shows that the tablet releases $50 \%$ and $60 \%$ of drug after 5 and $6 \mathrm{~h}$ of dissolution, respectively. Moreover, the percentage of drug released immediately after the neutralization step in the unconventional dissolution method is considerably higher than in the conventional one.

\section{Testing of Enteric Tablets by Varying pH}

A comparison of the two release profiles in Figures 1 and 2 shows that the first step to guarantee the desired release profile is to choose a polymer that is characterized by an enteric behavior even if drug administration happens in fed conditions. For this purpose, a pH-sensitive MMA/ AA copolymer for the enteric release was previously developed (23). Tablets constituted from this copolymer and diclofenac $(75 / 25 \mathrm{w} / \mathrm{w})$ were tested according to both the conventional dissolution method, which is characterized by a constant $\mathrm{pH}$ value of 1 in the first stage, and the modified $\mathrm{pH}$ evolution in the gastrointestinal tract described above, in which the $\mathrm{pH}$ decreases from 4.8 during the first stage. In this case, a three-stage $\mathrm{pH}$ 
evolution was adopted for both dissolution methods to simulate the passage in the jejunum, which is characterized by a $\mathrm{pH}$ value of 7.4. The obtained release profiles are shown in Figure 4, on the right axis. In this case, the comparison between the release patterns during the first stage of dissolution (two hours) shows that the release is not substantially affected by $\mathrm{pH}$ evolution. In fact, the release obtained according to the conventional dissolution pattern, characterized by $\mathrm{pH} 1$ (solid diamonds in Figure 4), is negligible during the first two hours, although the release profile obtained under the modified dissolution method (empty diamonds in Figure 4 ) is very low during the first step $(<3 \%$ drug is released in this stage). In the second stage, when the dissolution medium was neutralized, the release increased gradually during the 20 min following the neutralization for the tablet tested according to the conventional dissolution method, with a slow increment of drug release during the time. Whereas, for the tablet tested under a variation of $\mathrm{pH}$ in the dissolution medium, there was a sudden increase in the drug released a few minutes after the neutralization (empty diamonds in Figure 4). Despite these different behaviors, the percentages of drug released for both cases are very similar for long dissolution times. The release from the tablets tested under both conventional and modified $\mathrm{pH}$ conditions continued to increase very slowly, showing a prolonged release behavior. For these pharmaceutical formulations, the percentage of drug released after $5 \mathrm{~h}$ of dissolution was less than $15 \%$ for the tablets analyzed according to both conventional dissolution method and the unconventional one. This result is due to the composition of the tablets, which were formulated by mixing a large amount of polymer that significantly delays the release kinetics. The tested copolymer is not influenced by the $\mathrm{pH}$ history in the gastrointestinal tract, and the release kinetics is not affected by the $\mathrm{pH}$ values of the dissolution medium, which means that the tablet behavior is constant and predictable in the gastrointestinal tract. Because of their properties, the use of these copolymers is especially suitable for oral delivery purposes.

\section{Testing of Commercial Tablets in the Simulated Stomach}

Once the influence of the $\mathrm{pH}$ profile on the release pattern was evaluated and an enteric copolymer that could minimize the negative influence of the $\mathrm{pH}$ profile on drug release was prepared, the effect of the mechanical conditions in the stomach on the release was studied. The hydrodynamics (and thus the mixing conditions) of the real stomach is far from the one realized in USP Apparatus 2. Impulsive forces and fluid resistance due to

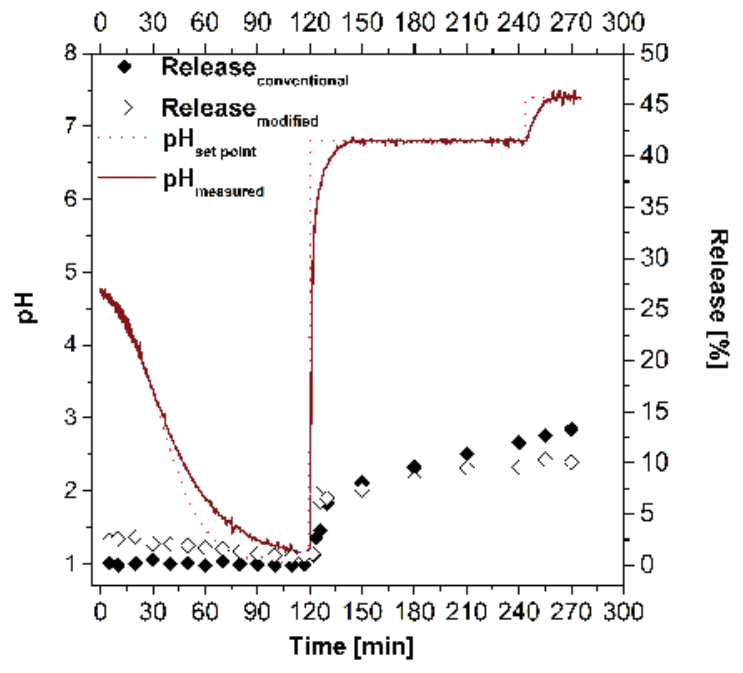

Figure 4. Three step $\mathrm{pH}$ evolution into the gastrointestinal tract on left axis (the red dotted line is the set point curve and the continuous line is the measured one). The drug release patterns from the enteric copolymer matrices according to the conventional ( $\$$ ) and modified $(\diamond) \mathrm{pH}$ evolution on the right axis.

mixing are higher in the gastrointestinal tract than in the conventional apparatus (29). In particular, the stomach is not perfectly mixed, and the drug concentration can vary along the stomach length (30). Thus, the release from the commercial diclofenac extended-release tablets was analyzed using the proposed in vitro device. During the dissolution test, samples of the dissolution medium were withdrawn from both the bottom and the top of the artificial stomach to evaluate the effects of shape and mixing on the drug release patterns. During the first two hours, 16 samples were withdrawn every 15 min from both the bottom and the top, with a volume decrease that is negligible with respect to the total dissolution volume. A comparison of the drug concentration at the top and the bottom of the device was possible to evaluate the mixing of the dissolution medium and the amount of drug that diffuses into the stomach. Figure 5 shows a comparison of drug release obtained using the artificial stomach with that obtained from the conventional dissolution method. In particular, during the first two hours of dissolution, the drug concentration at the bottom of the device where the dissolving tablet is located suddenly increases from zero (solid black circles), then decreases. This is the result of a dilution with the dissolution medium contained in the upper part of the stomach, which is due to both diffusion and mixing effects caused by agitation. Subsequently, the concentration increases again, due to the effect of the tablet dissolution. On the other hand, looking at the release at the top of the stomach (solid gray circles in Figure 5), it slightly increases from zero to a very small value, meaning that the mixing condition does not affect the entire stomach length. After the stomach stage, the 


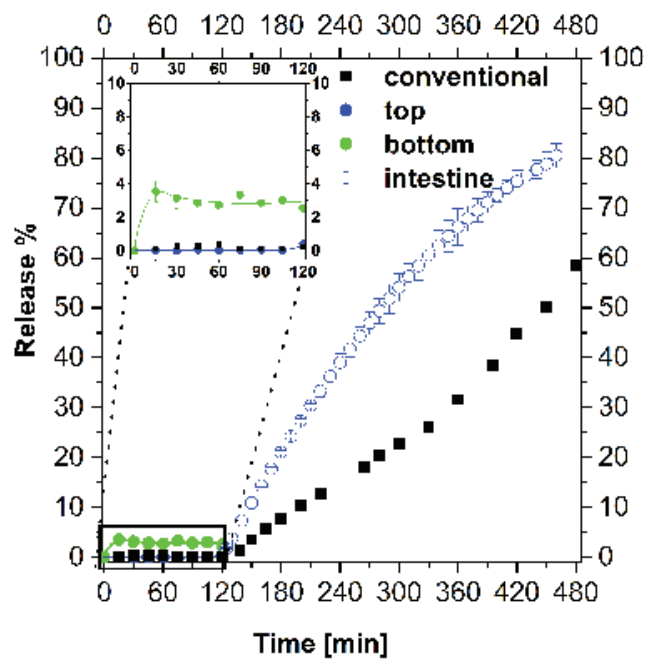

Figure 5. Drug release from a commercial tablet of diclofenac both

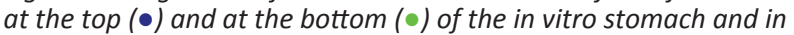
the intestine (०). The release pattern is compared with the ones obtained according to the conventional dissolution method (घ).

The inset represents a magnification of the first $120 \mathrm{~min}$ of dissolution.

release is evaluated quantitatively upon moving the stomach contents to Apparatus 2 and neutralizing it to simulate its passage into the intestine. Release in the intestine is shown in Figure 5 as empty circles. When the stomach contents were transferred to the USP vessel, the dissolution medium was homogenized and the release started from a value that is intermediate between the bottom and the top of the stomach. The release was faster for the tablet subjected to the dissolution conditions reproduced in the artificial stomach than for the one subjected to conventional conditions. The difference was caused by the higher shear experienced by the tablet in the artificial stomach that mechanically ruins the tablet during the first stage, causing a higher drug release.

\section{CONCLUSIONS}

This work evaluated the influence of two of the main physiological factors in the gastrointestinal tract affected by food intake, evolution of $\mathrm{pH}$ and mixing conditions in the stomach. Release profiles from a commercial diclofenac tablet were evaluated according to both the conventional dissolution method and a modified method that takes into account the real pH profile in fed conditions. The tablets behaved differently because of partial dissolution of the coating due to the high $\mathrm{pH}$ values in the first stage of the modified method. To overcome these issues leading to an undesired release pattern, tablets based on an enteric copolymer were tested and showed a release pattern not influenced by the variations of the $\mathrm{pH}$ profile between the fasted and fed conditions. Moreover, the commercial tablets were tested using an in vitro device able to reproduce the mechanical conditions of the stomach, to evaluate the effect of the mixing conditions on drug release. Again, the release pattern was strongly influenced by the different hydrodynamic conditions.

This work showed the effects of the chemical and mechanical reproduction of gastrointestinal behavior on pharmaceuticals, with the aim of improving dissolution test reliability when a pharmaceutical form is tested. Once all the involved phenomena are correctly identified and comprehended, a combined approach that accounts for both the chemical ( $\mathrm{pH}$ conditions) and mechanical (peristaltic contractions) behaviors, gastric emptying, and enzyme secretion is necessary to more closely reproduce a drug's fate.

\section{ACKNOWLEDGMENT}

The economic contribution of Ministero dell'Istruzione dell'Università e della Ricerca (contracts grant number PRIN 2010-11-20109PLMH2) is gratefully acknowledged.

\section{CONFLICT OF INTEREST}

The authors declare that there is no conflict of interest.

\section{REFERENCES}

1. Toothaker, R. D.; Welling, P. G. The Effect of Food on Drug Bioavailability. Annu. Rev. Pharmacol. Toxicol. 1980, 20 (1), 173-199. DOI:10.1146/annurev. pa.20.040180.001133.

2. Welling, P. G. Influence of food and diet on gastrointestinal drug absorption: A review. J. Pharmacokinet. Biopharm. 1977, 5 (4), 291-334. DOI: 10.1007/BF01061694.

3. Varum, F. J. O.; Hatton, G. B.; Basit, A. W. Food, physiology and drug delivery. Int. J. Pharm. 2013, 457 (2), 446-460. DOI: 10.1016/j.ijpharm.2013.04.034.

4. Oral Controlled Release Formulation Design and Drug Delivery: Theory to Practice. Wen, H., Park, K., Eds.; John Wiley: Hoboken, NJ, 2010.

5. Trystram, G. Modelling of food and food processes. J. Food Eng. 2012, 110 (2), 269-277. DOI: 10.1016/j. jfoodeng.2011.05.001.

6. Ferrua, M. J.; Singh, R. P. Modeling the Fluid Dynamics in a Human Stomach to Gain Insight of Food Digestion. J. Food Sci. 2010, 75 (7), R151-R162. DOI: 10.1111/j.1750-3841.2010.01748.x.

7. Marra, F.; Ferrua, M. J.; Singh, R. P. Experimental characterization of the fluid dynamics in an in-vitro system simulating the peristaltic movement of the stomach wall. Procedia Food Sci. 2011, 1, 1473-1478. DOI: 10.1016/j.profoo.2011.09.218.

8. Harrison, S. M.; Cleary, P. W. Towards modelling of fluid flow and food breakage by the teeth in the oral 
cavity using smoothed particle hydrodynamics (SPH). Eur. Food Res. Technol. 2014, 238 (2), 185-215. DOI: 10.1007/s00217-013-2077-8.

9. Van Wey, A. S.; Cookson, A. L.; Roy, N. C.; McNabb, W. C.; Soboleva, T. K.; Wieliczko, R. J.; Shorten, P. R. A mathematical model of the effect of $\mathrm{pH}$ and food matrix composition on fluid transport into foods: An application in gastric digestion and cheese brining. Food Res. Int. 2014, 57, 34-43. DOI: 10.1016/j. foodres.2014.01.002.

10. Ho, Q. T.; Carmeliet, J.; Datta, A. K.; Defraeye, T.; Delele, M. A.; Herremans, E.; Opara, L.; Ramon, H.; Tijskens, E.; van der Sman, R.; Van Liedekerke, P.; Verboven, P.; Nicolai, B. M. Multiscale modeling in food engineering. J. Food Eng. 2013, 114 (3), 279291. DOI: 10.1016/j.jfoodeng.2012.08.019.

11. Gulati, T.; Datta, A. K. Enabling computer-aided food process engineering: Property estimation equations for transport phenomena-based models. J. Food Eng. 2013, 116 (2), 483-504. DOI: 10.1016/j. jfoodeng.2012.12.016.

12. Hur, S. J.; Lim, B. O.; Decker, E. A.; McClements, D. J. In vitro human digestion models for food applications. Food Chem. 2011, 125 (1), 1-12. DOI: 10.1016/j. foodchem.2010.08.036.

13. Boisen, S.; Eggum, B. O. Critical Evaluation of in Vitro Methods for Estimating Digestibility in SimpleStomach Animals. Nutr. Res. Rev. 1991, 4 (1), 141162. DOI: $10.1079 /$ NRR19910012.

14. Sagum, R.; Arcot, J. Effect of domestic processing methods on the starch, non-starch polysaccharides and in vitro starch and protein digestibility of three varieties of rice with varying levels of amylose. Food Chem. 2000, 70 (1), 107-111. DOI: 10.1016/S03088146(00)00041-8.

15. Chen, J.; Gaikwad, V.; Holmes, M.; Murray, B.; Povey, M.; Wang, Y.; Zhang, Y. Development of a simple model device for in vitro gastric digestion investigation. Food Funct. 2011, 2 (3-4), 174-182. DOI: 10.1039/COFO00159G.

16. Tharakan, A.; Norton, I. T.; Fryer, P. J.; Bakalis, S. Mass Transfer and Nutrient Absorption in a Simulated Model of Small Intestine. J. Food Sci. 2010, 75 (6), E339-E346. DOI: 10.1111/j.1750-3841.2010.01659.x.

17. Minekus, M.; Marteau, P.; Havenaar, R.; Huis in't Veldr, J. H. J. A Multicompartmental Dynamic Computercontrolled Model Simulating the Stomach and Small Intestine. Altern. Lab. Anim. 1995, 23 (2), 197-209.

18. Wickham, M.; Faulks, R. Dynamic Gastric Model. U.S. Patent 8,092,222, Jan 30, 2008.

19. Kong, F.; Singh, R. P. A Human Gastric Simulator (HGS) to Study Food Digestion in Human Stomach. J. Food Sci. 2010, 75 (9), E627-E635. DOI: 10.1111/j.1750-3841.2010.01856.x

20. Bornhorst, G. M.; Singh, R. P. Gastric Digestion In Vivo and In Vitro: How the Structural Aspects of Food Influence the Digestion Process. Food Sci. Technol. 2014, 5, 111-132. DOI: 10.1146/annurevfood-030713-092346.

21. Li, Y.; Hu, M.; McClements, D. J. Factors affecting lipase digestibility of emulsified lipids using an in vitro digestion model: Proposal for a standardised $\mathrm{pH}$-stat method. Food Chem. 2011, 126 (2), 498-505. DOI: 10.1016/j.foodchem.2010.11.027.

22. Nokhodchi, A.; Asare-Addo, K. Drug release from matrix tablets: physiological parameters and the effect of food. Expert Opin. Drug Delivery 2014, 11 (9), 1401-1418. DOI: 10.1517/17425247.2014.924498

23. Barba, A. A.; Dalmoro, A.; De Santis, F.; Lamberti, G. Synthesis and characterization of P(MMA-AA) copolymers for targeted oral drug delivery. Polym. Bull. 2009, 62 (5), 679-688. DOI: 10.1007/s00289009-0040-4.

24. <711> Dissolution. In The United States Pharmacopeia and National Formulary USP 28-NF 23; The United States Pharmacopeial Convention, Inc.: Rockville, MD, 2005; pp2412-2414.

25. Kukura, J.; Baxter, J. L.; Muzzio, F. J. Shear distribution and variability in the USP Apparatus 2 under turbulent conditions. Int. J. Pharm. 2004, 279 (1-2), 9-17. DOI: 10.1016/j.ijpharm.2004.03.033.

26. Mudie, D. M.; Amidon, G. L.; Amidon, G. E. Physiological Parameters for Oral Delivery and in Vitro Testing. Mol. Pharmaceutics 2010, 7 (5), 1388-1405. DOI: 10.1021/mp100149j.

27. Marteau, P.; Flourie, B.; Pochart, P.; Chastang, C.; Desjeux, J.-F.; Rambaud, J.-C. Effect of the microbial lactase (EC 3.2.1.23) activity in yoghurt on the intestinal absorption of lactose: An in vivo study in lactase-deficient humans. Br. J. Nutr. 1990, 64 (1), 71-79. DOI: 10.1079/BJN19900010.

28. Cascone, S.; De Santis, F.; Lamberti, G.; Titomanlio, G. The influence of dissolution conditions on the drug ADME phenomena. Eur. J. Pharm. Biopharm. 2011, 79 (2), 382-391. DOI: 10.1016/j.ejpb.2011.04.003.

29. Kamba, M.; Seta, Y.; Takeda, N.; Hamaura, T.; Kusai, A.; Nakane, H.; Nishimura, K. Measurement of agitation force in dissolution test and mechanical destructive force in disintegration test. Int. J Pharm. 2003, 250 (1), 99-109. DOI: 10.1016/S0378-5173(02)00535-5.

30. Pal, A.; Brasseur, J. G.; Abrahamsson, B. A stomach road or "Magenstrasse" for gastric emptying. J. Biomech. 2007, 40 (6), 1202-1210. DOI: 10.1016/j. jbiomech.2006.06.006. 